\title{
Quantum Scalar Field Behavior of the Conformally Flat Part of the S-K-S Metric
}

\author{
H. A. Camargo ${ }^{1}$, E. J. Gonzalez de Urreta ${ }^{2,3}$ and M. Socolovsky ${ }^{2,4^{*}}$ \\ ${ }^{1}$ Facultad de Ciencias, Universidad Nacional Autónoma de México \\ Circuito Exterior, Ciudad Universitaria, 04510, México D.F. \\ ${ }^{2}$ Instituto de Ciencias, Universidad Nacional de General Sarmiento \\ Juan María Gutiérrez 1150 (B1613GSX), Los Polvorines, Pcia. de Buenos Aires, Argentina \\ ${ }^{3}$ Posdoctoral fellowship, CONICET, Argentina \\ ${ }^{4}$ With a leave of abscence from Instituto de Ciencias Nucleares, UNAM, México \\ Email: msocolov@ungs.edu.ar
}

\begin{abstract}
Using Weyl geometry, we study some properties of the scalar $\phi$-field representing the conformally flat part of the Schwarzschild-Kruskal-Szekeres (S-K-S) metric in the black hole region. As expected, the classical field diverges (logarithmically) at the singularity, where it is infinitely heavy, but remains finite at the horizons, where it becomes massless. The quantum propagator in the radial direction is also computed, exhibiting rapid oscillations and a divergent absolute value at the singularity.
\end{abstract}

Keywords: Schwarzschild black hole, scalar field, quantization.

\section{Introduction}

It is well known that a Weyl geometry [1] with vanishing or flat 1-form (integrable Weyl geometry) is equivalent to a Riemannian geometry. This equivalence allows us to interpret a conformally flat metric as a massless scalar field $\phi$ in Minkowski spacetime [2,3]. Examples of the use of this equivalence include the study of the quantum fluctuations of the four-dimensional anti-De Sitter spacetime, with interesting results [3]. In the present paper, we apply the aforementioned equivalence to the 2-dimensional conformally flat part of the Schwarzschild-Kruskal-Szekeres (S-K-S) $[4,5,6]$ metric. Classically the field $\phi$ diverges logarithmically at the singularity, remaining finite at the horizons. Eliminating, through a change of coordinates, the time variable $(t)$ in favor of the Schwarzschild radial coordinate $(r)$, allows us to integrate over the spatial coordinate $(x)$ in the action for the the field $\phi$, leading to an effective 1-dimensional action density corresponding to a non-relativistic particle with $r$-dependent mass, infinite at the singularity and zero at the horizons, with a linear potential proportional to $\phi$. The quantum propagator represented by a Feynman path integral [7] with quadratic integrand can be computed exactly using standard techniques [8], which exhibits strong oscillations and a divergent absolute value at the singularity.

The result, incomplete as it stands since it is two-dimensional instead of four-dimensional -it ignores the angular variables-, suggests that the behavior of a true quantum theory of black holes would exhibit, at least, large fluctuations near or at the classical singularity. It is not clear how the non-conformal part of the metric could totally suppress the infinite divergences found here. In any case, the attempt of this paper is to taste the quantum behavior of the metric at the singularity.

We use the Natural System of units: $c=G=\hbar=1$.

\section{$2 \quad$ Weyl and Riemann Geometries}

Weyl geometry, which originated as an attempt to unify Einstein general relativity with electromagnetism, is the generalization of Riemannian geometry in which lengths of vectors $L=\|V\|^{2}$ are allowed to change by path dependent scale factors $s=e^{\int_{c} W}$ after parallel transport along smooth curves $c$ : $L \rightarrow L^{\prime}=s L . W$ is the Weyl field transforming as a 1-form under local coordinates transformations. This is equivalent to the statement that the covariant derivative of the metric with respect to the Weyl 
connection $\left(\Gamma_{W}\right)_{\nu \rho}^{\mu}=\left(\Gamma_{L C}\right)_{\nu \rho}^{\mu}+W_{\nu \rho}^{\mu}$ is given by $D_{\mu}^{(W)} g_{\nu \lambda}=-W_{\mu} g_{\nu \lambda}$, where $\left(\Gamma_{L C}\right)_{\nu \rho}^{\mu}$ is the Levi-Civita connection and $W_{\nu \rho}^{\mu}=\frac{1}{2}\left(\delta_{\nu}^{\mu} W_{\rho}+\delta_{\rho}^{\mu} W_{\nu}-g_{\nu \rho} g^{\mu \lambda} W_{\lambda}\right)$ is the Weyl tensor. Instead, in Riemannian geometry, vector fields can only rotate after parallel displacement, and $D_{\mu}^{(L C)} g_{\nu \lambda}=0$.

As mentioned in the Introduction, any Riemannian manifold $\left(M_{\mathcal{R}}^{n}, g_{\mu \nu}\right)$ can be identified with a Weyl manifold $[1,2,3]\left(M_{\mathcal{W}}^{n}, g_{\mu \nu}, W\right)$ with zero Weyl field $W=W_{\mu} \mathrm{d} x^{\mu}$ or with zero gauge curvature i.e. $W=d \alpha$ with $\alpha$ a smooth function. Then, in particular,

$$
\left(M_{\mathcal{R}}^{n}, g_{\mu \nu}\right) \equiv\left(M_{\mathcal{W}}^{n}, g_{\mu \nu}, 0\right)
$$

with $M_{\mathcal{R}}^{n}=M_{\mathcal{W}}^{n}=M^{n}$ as differentiable manifolds, since the differentiable structure is a substratum of the metric structure. After a Weyl symmetry transformation (invariance of $L^{\prime}$ )

$$
g_{\mu \nu} \rightarrow g_{\mu \nu}^{\prime}=\lambda^{-1} g_{\mu \nu}, W \rightarrow W^{\prime}=W-\lambda^{-1} \mathrm{~d} \lambda
$$

where $\lambda$ is a smooth positive function, $\lambda^{-1} g_{\mu \nu}$ a conformal change of the metric, and $W-\lambda^{-1} \mathrm{~d} \lambda$ a gauge transformation of the Weyl field, one has

$$
\left(M^{n}, g_{\mu \nu}, 0\right) \sim\left(M^{n}, \lambda^{-1} g_{\mu \nu},-\lambda^{-1} \mathrm{~d} \lambda\right),
$$

where $\sim$ denotes equivalence within the Weyl class $\left[\left(M^{n}, g_{\mu \nu}, 0\right)\right]$. In particular, when

$$
g_{\mu \nu}=e^{\phi} \eta_{\mu \nu}
$$

where $\phi$ is a real function of the $x^{\mu}$ 's and $\eta_{\mu \nu}=\operatorname{diag}(1,-1, \cdots,-1)$ is the $n$-dimensional Minkowski metric, the choice

$$
\lambda=e^{\phi}
$$

leads to

$$
\left(M^{n}, e^{\phi} \eta_{\mu \nu}, 0\right) \sim\left(M^{n}, \eta_{\mu \nu},-\mathrm{d} \phi\right)
$$

i.e. $W=\mathrm{d} \phi$. Since $d^{2}=0$ the r.h.s. of (6) has zero gauge curvature and therefore

$$
\left(M^{n}, e^{\phi} \eta_{\mu \nu}\right) \equiv\left(M^{n}, \eta_{\mu \nu},-\mathrm{d} \phi\right)
$$

I.e., classically, the whole gravitational information in $\left(M^{n}, e^{\phi} \eta_{\mu \nu}\right)$ is encoded in the field $\phi$ living in Minkowski space. If the equation of motion of the $\phi$-field admits a Lagrangian, then the corresponding quantum theory can, in principle, be constructed via the Feynman path integral method.

\section{S-K-S Metric}

The maximal analytic extension of the Schwarzschild metric is the Schwarzschild-Kruskal-Szekeres metric $[4,5,6]$ given by

$$
\mathrm{d} s^{2}(t, x, \theta, \varphi)=\frac{8 M}{r} e^{-\frac{r}{2 M}}\left(\mathrm{~d} t^{2}-\mathrm{d} x^{2}\right)-r^{2} \mathrm{~d} \Omega^{2}
$$

where $2 M$, the Schwarzschild radius, is the position of the future and past horizons, $\mathrm{d} \Omega^{2}=\mathrm{d} \theta^{2}+\sin ^{2} \theta \mathrm{d} \varphi^{2}$, $t \in(-\infty,+\infty), x \in(-\infty,+\infty),[t]=[x]=[r]=[$ length], $r>0$ (the singularities, future and past) depends implicitly on $t$ and $x$ i.e. $r=r(t, x)$ through

$$
x^{2}-t^{2}=4 M^{2}\left(\frac{r}{2 M}-1\right) e^{r / 2 M} .
$$

(In the usual dimensionless coordinates $U=x / 2 M$ and $V=t / 2 M$ the factor $4 M^{2}$ in the r.h.s. of (9) would be abscent.)

As is well known, the Kruskal-Szekeres coordinates are usually defined through a change of variables involving the Eddington-Finkelstein coordinates. Physically, the K-S coordinates are locally inertial coordinates at the future and past horizons of the Schwarzschild black hole. They can also be thought as natural affine parameters for the generators of such horizons [9]. 
The Kruskal diagram of the S-K-S metric is exhibited in Fig. 2. The regions above and below the two horizons $r=2 M$ are, respectively, the black hole and white hole regions, while the right and left wedges are the asymptotically flat $(r \rightarrow \infty)$ regions. (Since we are interested in the black hole we omit in the diagram the symmetric singularity in the white hole, also at $\mathrm{r}=0$.)

The conformally flat part of the metric is

$$
\mathrm{d} s_{c f}^{2}=e^{\phi}\left(\mathrm{d} t^{2}-\mathrm{d} x^{2}\right),
$$

where $\phi$ is a classical 2-dimensional field in Minkowski space $(t, x)$ given by

$$
\phi=\phi(r)=\ln \left(\frac{8 M}{r}\right)-\frac{r}{2 M} .
$$

Clearly, $\phi$ diverges logarithmically $(\phi \rightarrow+\infty)$ as $r \rightarrow 0$ and linearly $(\phi \rightarrow-\infty)$ as $r \rightarrow \infty$ (asymptotically flat regions), at the horizons $\phi(2 M)=2 \ln 2-1$,

$$
\phi^{\prime}(r)=\frac{\mathrm{d} \phi(r)}{\mathrm{d} r}=-\left(\frac{1}{r}+\frac{1}{2 M}\right)<0,
$$

and $\phi^{\prime \prime}(r)=1 / r^{2}$. (See Fig. 1.)

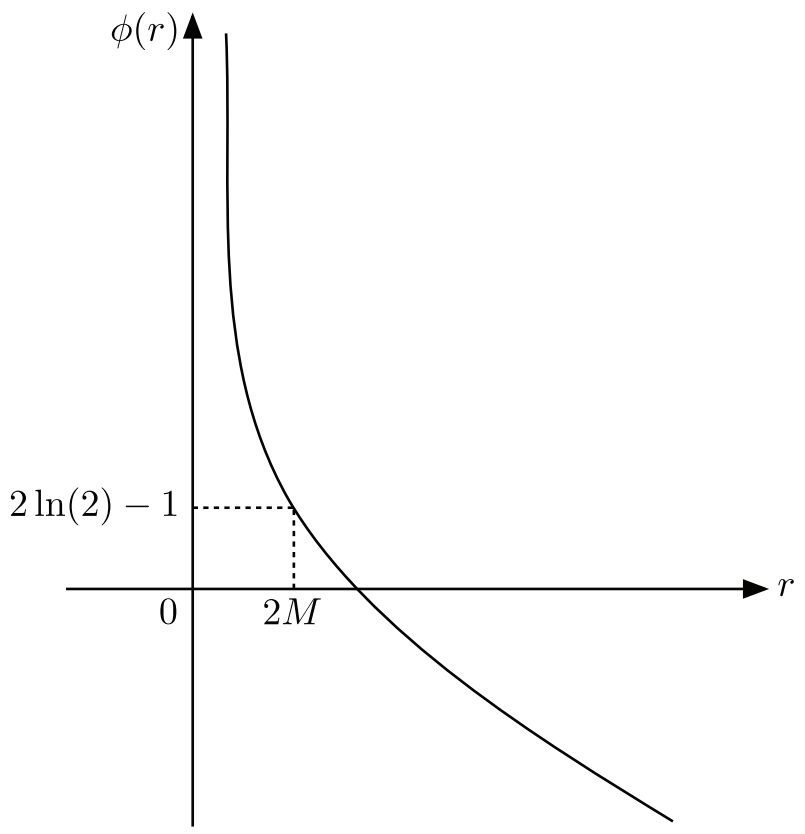

Figure 1. Classical field $\phi$ in 2-dimensional Minkowski space.

In the black hole (B.H.) region, $t \in[0,+\infty)$ and $x \in(-\infty,+\infty)$; for each $x, r \in[2 M, 0)$ and

$$
t=t(x, r)=\sqrt{f(r)+x^{2}}
$$

with

$$
f(r)=4 M^{2}\left(1-\frac{r}{2 M}\right) e^{r / 2 M} .
$$

Also, $t(x, 2 M)=|x|$ and $\lim _{r \rightarrow 0} t(x, r)=\sqrt{x^{2}+4 M^{2}}$. (See Fig. 2.)

Using

$$
\frac{\partial \phi}{\partial t}=\frac{\mathrm{d} \phi}{\mathrm{d} r} \frac{\partial r}{\partial t}, \frac{\partial \phi}{\partial x}=\frac{\mathrm{d} \phi}{\mathrm{d} r} \frac{\partial r}{\partial x}, \frac{\partial^{2} \phi}{\partial t^{2}}=\frac{d^{2} \phi}{\mathrm{d} r^{2}}\left(\frac{\partial r}{\partial t}\right)^{2}+\frac{\mathrm{d} \phi}{\mathrm{d} r}\left(\frac{\partial^{2} r}{\partial t^{2}}\right), \frac{\partial^{2} \phi}{\partial x^{2}}=\frac{d^{2} \phi}{\mathrm{d} r^{2}}\left(\frac{\partial r}{\partial x}\right)^{2}+\frac{\mathrm{d} \phi}{\mathrm{d} r}\left(\frac{\partial^{2} r}{\partial x^{2}}\right)
$$




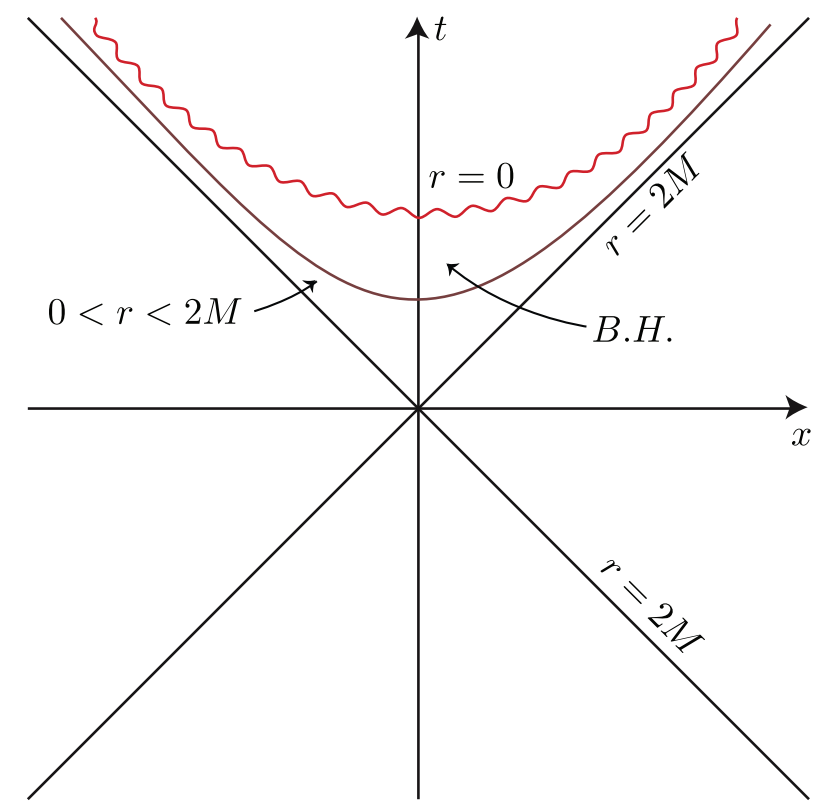

Figure 2. Black hole region in the S-K-S metric.

and, through the use of the implicit function theorem,

$$
\begin{gathered}
\frac{\partial r}{\partial t}=-\frac{\frac{\partial F}{\partial t}}{\frac{\partial F}{\partial r}}=-\frac{2 t}{r} e^{-r / 2 M}, \frac{\partial r}{\partial x}=-\frac{\frac{\partial F}{\partial x}}{\frac{\partial F}{\partial r}}=\frac{2 x}{r} e^{-r / 2 M} \\
\frac{\partial^{2} r}{\partial t^{2}}=-\frac{2}{r} e^{-r / 2 M}-\frac{4 t^{2}}{r^{3}} e^{-r / M}\left(1+\frac{r}{2 M}\right), \frac{\partial^{2} r}{\partial x^{2}}=\frac{2}{r} e^{-r / 2 M}-\frac{4 x^{2}}{r^{3}} e^{-r / M}\left(1+\frac{r}{2 M}\right),
\end{gathered}
$$

where

$$
F(t, x, r):=t^{2}-x^{2}-f(r)=0,
$$

the wave equation for the $\phi$-field is

$$
\frac{\partial^{2} \phi}{\partial t^{2}}-\frac{\partial^{2} \phi}{\partial x^{2}}=\Phi(r)
$$

where

$$
\Phi(r)=\frac{32 M^{2}}{r^{4}} e^{-r / 2 M}
$$

is a source for $\phi . \Phi \rightarrow+\infty$ as $r \rightarrow 0$ and $\Phi(2 M)=\frac{2}{M^{2} e}$. (See Fig. 3.)

It is clear that the equation satisfied by $\phi$ is not unique, but once the differential operator in the l.h.s. of (18) is chosen -in this case the d'Alembertian in two-dimensional Minkowski spacetime- the source term in the r.h.s. appears automatically as a consequence of the S-K-S metric (8) which is a solution of Einstein equations in four dimensions. In two spacetime dimensions Einstein relativity is trivial and in alternative theories of gravity like that in ref. [10], the equation for a conformally flat metric $e^{\varphi} \eta_{\mu \nu}$ in vacuum and without cosmological constant is $\left(\frac{\partial^{2}}{\partial t^{2}}-\frac{\partial^{2}}{\partial x^{2}}\right) \varphi=0$ [2].

It is easily verified that (18) can be derived from the Lagrangian density

$$
\mathcal{L}_{B H}=\frac{1}{2}\left(\eta^{\mu \nu}\left(\partial_{\mu} \phi\right)\left(\partial_{\nu} \phi\right)+2 \Phi \phi\right)
$$

with $\eta^{\mu \nu}=\operatorname{diag}(1,-1), \mu, \nu=t, x$. There is the corresponding action

$$
S_{B H}=\int_{-\infty}^{+\infty} \mathrm{d} x \int_{x}^{\sqrt{x^{2}+4 M^{2}}} \mathrm{~d} t \mathcal{L}_{B H}=\frac{1}{2} \int_{-\infty}^{+\infty} \mathrm{d} x \int_{x}^{\sqrt{x^{2}+4 M^{2}}} \mathrm{~d} t\left(\left(\frac{\partial \phi}{\partial t}\right)^{2}-\left(\frac{\partial \phi}{\partial x}\right)^{2}+\frac{64 M^{2}}{r^{4}} e^{-r / 2 M} \phi\right)
$$




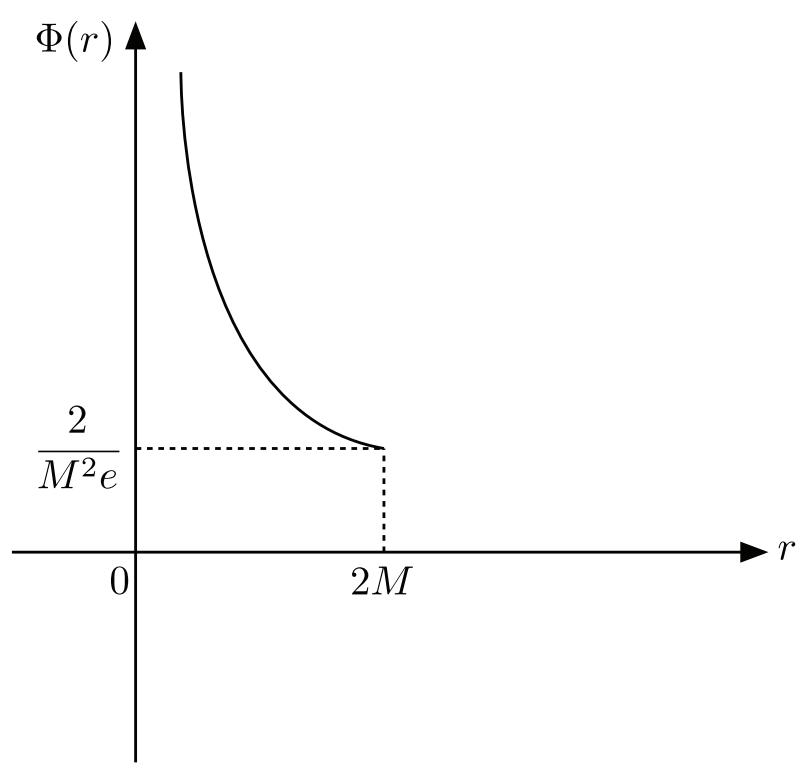

Figure 3. Source of the $\phi$-field in the BH region.

which, after the change of variables $(x, t) \rightarrow(x, r)$ with Jacobian $\frac{\partial(x, r)}{\partial(x, t)}=-\frac{2 t}{r} e^{-r / 2 M}$ becomes

$$
S_{B H}=4 M^{2} \int_{0}^{2 M} \mathrm{~d} r \int_{-\infty}^{+\infty} \frac{\mathrm{d} x}{\sqrt{f(r)+x^{2}}} \frac{1}{r}\left(\left(1-\frac{r}{2 M}\right)\left(\phi^{\prime}(r)\right)^{2}+\left(\frac{2}{r}\right)^{2} \phi\right) .
$$

The $x$-integration can be done explicitly, though it gives the infinite factor (logarithmic divergence) $\int_{-\infty}^{+\infty} \frac{\mathrm{d} x}{\sqrt{f(r)+x^{2}}}=\lim _{N \rightarrow \infty} \int_{-N}^{+N} \frac{\mathrm{d} y}{\sqrt{1+y^{2}}}=2 \lim _{N \rightarrow \infty} \ln N=+\infty$. Since we can approach the singularity $r=0$ at any fixed value of the spatial coordinate $x$, and since the purpose is to compute the quantum propagator from the singularity to any point within or at the horizon, we factor out this infinity and define an $r$-regulated effective action "density"

$$
\begin{gathered}
s_{B H}=\lim _{\epsilon \rightarrow 0} s_{B H \epsilon}, \\
s_{B H \epsilon}=4 M^{2} \int_{\epsilon}^{2 M} \frac{\mathrm{d} r}{r}\left(\left(1-\frac{r}{2 M}\right)\left(\phi^{\prime}(r)\right)^{2}+\left(\frac{2}{r}\right)^{2} \phi(r)\right) .
\end{gathered}
$$

The quantum propagator from the (divergent) configuration $\phi_{1}=\phi(\epsilon)=\ln \left(\frac{8 M}{\epsilon}\right)-\frac{\epsilon}{2 M}$ to $\phi_{2}=$ $\phi(2 M)=2 \ln 2-1$ is given by the path integral [7]

$$
<\phi_{2} \mid \phi_{1}>_{\epsilon}=\int_{\phi_{1}}^{\phi_{2}} \mathcal{D} \phi e^{4 i M^{2} \int_{\epsilon}^{2 M} \frac{\mathrm{d} r}{r}\left(\left(1-\frac{r}{2 M}\right)\left(\phi^{\prime}(r)\right)^{2}+\left(\frac{2}{r}\right)^{2} \phi(r)\right)},
$$

which corresponds to the non-relativistic motion of a particle with an $r$ dependent effective mass

$$
m_{e f f}(r)=\frac{8 M^{2}}{r}\left(1-\frac{r}{2 M}\right)
$$

infinitely heavy at the singularity: $m_{e f f}(\epsilon) \sim \frac{1}{\epsilon} \rightarrow \infty$, and massless at the horizons: $m_{e f f}(2 M)=0$. (Reminding that within the horizons, $r$ behaves as a timelike coordinate $\tau$, the equivalent 1-dimensional Lagrangian would be $L=\frac{1}{2} m(\tau) \dot{q}(\tau)^{2}+\left(\frac{4 M}{\tau}\right)^{2} q(\tau)$.) is

The path integral, being quadratic, can be computed exactly by standard methods [8], and the result

$$
<\phi_{2}\left|\phi_{1}>_{\epsilon}=e^{i s_{B H}\left(\phi_{c l}\right)}<\phi=0,2 M\right| \phi=0, \epsilon>
$$


where $\phi_{c l}$ is given by (11),

$$
s_{B H \epsilon}\left(\phi_{c l}\right)=\frac{5}{2}-4 \ln 2-\left(\frac{1}{2}-4 \ln 2\right)\left(\frac{2 M}{\epsilon}\right)^{2}-\left(1-2\left(\frac{2 M}{\epsilon}\right)^{2}\right) \ln \left(\frac{2 M}{\epsilon}\right)-3\left(\frac{2 M}{\epsilon}\right)+\frac{\epsilon}{2 M},
$$

and

$$
<\phi=0,2 M \mid \phi=0, \epsilon>=\int_{y(\epsilon)=y(2 M)=0} \mathcal{D} y e^{\frac{i}{2} \int_{\epsilon}^{2 M} \mathrm{~d} r m_{e f f}(r)\left(y^{\prime}(r)\right)^{2}}=\sqrt{\frac{m_{e f f}(\epsilon)}{2 \pi i(2 M-\epsilon)}} .
$$

As $\epsilon \rightarrow 0$,

$$
<\phi_{2} \mid \phi_{1}>_{\epsilon} \simeq e^{2 i\left(\frac{2 M}{\epsilon}\right)^{2} \ln \left(\frac{2 M}{\epsilon}\right)} \sqrt{\frac{M}{\pi \epsilon}}(1-i),
$$

which strongly oscillates and diverges at the singularity. In absolute value,

$$
\left|<\phi_{1}\right| \phi_{2}>_{\epsilon} \mid \simeq \sqrt{\frac{2 M}{\pi \epsilon}} .
$$

\section{Final Comments}

Though using Weyl geometry only the conformally flat part of the S-K-S metric can be interpreted as a scalar field in Minkowski spacetime, and therefore can be easily quantized through standard methods e.g. the Feynman path integral, we do not expect that the non-conformal part of the metric -the 2-spheres $S^{2}(r)$ - for which the Weyl trick can not be applied, can smoothen the violent oscillations and divergence exhibited by the quantum behavior of the metric in the neighbourhood of the singularity. We agree of course that the present analysis is far from complete -only the time/radial part of the black hole geometry is considered-; however, at least it gives a feeling of what happens, at the quantum level, in the otherwise misterious -collapse to a point- classical behavior at the singularity.

Acknowledgments. M.S. thanks discussions with A. Cabo (ICIMAF, Cuba), and E. Eiroa (IAFE-UBACONICET, Argentina); also thanks IAFE for its hospitality.

\section{References}

1. H. Weyl, Gravitation und Elektrizität, Sitzungsber. d. Preuss-Akad. d. Wissensch. 465 (1918). English translation: L.O'Reifearteigh, The Dawning of Gauge Theory (Princeton Univ. Press, Princeton, 1997), 24-37.

2. C. Romero, J.B. Fonseca-Neto, and M.L. Pucheu, Conformally flat spacetimes and Weyl frames, Found. Phys. 42, 224 (2012); arXiv: 1101.5333v1 [gr-qc].

3. H.A. Camargo, E.J. Gonzalez de Urreta, and M. Socolovsky, Weyl geometry, anti-de Sitter space, and $\Phi^{4}$-theory, Eur. Phys. J. Plus 131, 172 (2016); arXiv: 1511.04403v1 [phys. gen-ph].

4. K. Schwarzschild, Uber das Gravitationsfeld eines Massenpunktes nach der Einsteinschen Theorie, Sitzber. Deut. Akad. Wiss. Berlin, Kl. Math.-Phys. Tech. 189-196 (1916). English translation: On the Gravitational Field of a Mass Point According to Einstein Theory, Gen. Rel. Grav. 35, 951-959 (2003).

5. M.D. Kruskal, Maximal Extension of Schwarzschild Metric, Phys. Rev. 119, 1743-1745 (1960).

6. G. Szekeres, On the singularities of a Riemannian manifold, Publ. Math. Debrecen 7, 285-301 (1960).

7. R.P. Feynman, and A.R. Hibbs, Quantum Mechanics and Path Integrals (McGraw-Hill, New York, 1965).

8. L.S. Schulman, Techniques and Applications of Path Integration (J. Wiley, New York, 1981), pp. 31-41.

9. A. Fabbri, and J. Navarro-Salas, Modeling Black Hole Evaporation (Imperial College Press, London, 2005), pp. 32-39.

10. A.E. Sikkema, and R.B. Mann, Gravitation and cosmology in $(1+1)$ dimensions, Class. Quantum Grav. 8, 219-235 (1991). 